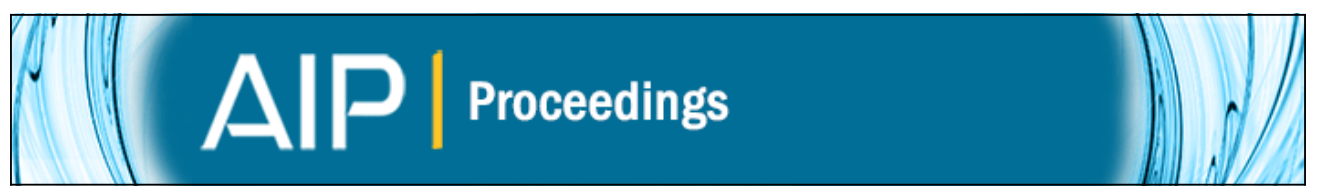

\title{
Wave Propagation in Water-saturated Sand and Grain Contact Physics
}

Nicholas P. Chotiros and Marcia J. Isakson

Citation: AIP Conference Proceedings 1272, 125 (2010); doi: 10.1063/1.3493053

View online: http://dx.doi.org/10.1063/1.3493053

View Table of Contents:

http://scitation.aip.org/content/aip/proceeding/aipcp/1272?ver=pdfcov

Published by the AIP Publishing

\section{Articles you may be interested in}

Confirmation of the Biot theory for water-saturated sands at high frequencies and effects of scattering on the attenuation of sound waves

J. Acoust. Soc. Am. 119, 709 (2006); 10.1121/1.2149770

An inversion for Biot parameters in water-saturated sand

J. Acoust. Soc. Am. 112, 1853 (2002); 10.1121/1.1511199

Synthetic array measurements of acoustical waves propagating into a water-saturated sandy bottom for a smoothed and a roughened interface

J. Acoust. Soc. Am. 107, 2329 (2000); 10.1121/1.428618

Response to: "Comments on 'Biot model of sound propagation in water-saturated sand'" [J. Acoust. Soc. Am. 103, 2723-2725 (1998)]

J. Acoust. Soc. Am. 103, 2726 (1998); 10.1121/1.422792

Comments on "Biot model of sound propagation in water-saturated sand" [J. Acoust. Soc. Am. 97, 199-214 (1995)]

J. Acoust. Soc. Am. 103, 2723 (1998); 10.1121/1.422791 


\title{
Wave Propagation in Water-saturated Sand and Grain Contact Physics
}

\author{
Nicholas P. Chotiros and Marcia J. Isakson \\ Applied Research Laboratories, The University of Texas at Austin, Texas 78713-8029
}

\begin{abstract}
Measurements in sandy ocean sediments over a broad range of frequencies show that the sound speed dispersion is significantly greater than that predicted by the Biot-Stoll model with constant coefficients, and the observed sound attenuation does not seem to follow a consistent power law. The observations may be explained in terms of the Biot-Stoll model with frequency-dependent complex frame bulk and shear moduli that are governed by the grain-grain contact physics, and random motion at the grain level. In the case of water-saturated sands, the contact stiffness is dominated by squirt flow and viscous drag of a thin fluid film that permeates the contact area. Using this approach, the observed sound and shear wave speeds and attenuations may be modeled over a broad band of frequencies.
\end{abstract}

Keywords: underwater, sediment, poroelastic, dispersion.

PACS: 43.30.Ma, 43.30.Pc, 43.30.Vh

\section{INTRODUCTION}

The ocean sediment is often approximated as a lossy fluid or a viscoelastic solid. These approximations are relatively simple to model with the tools that are currently available. Measurements have shown that there are significant discrepancies between these approximations and real ocean sediments. This study is particularly concerned with sandy ocean sediments. Physically, it is a granular material that is saturated with sea water. It is a two-phase medium in which the sand grains form a loose skeletal frame permeated by sea water. The logical starting point would be a poroelastic model, such as the Biot model [1], which was reformulated by Stoll and applied to sandy sediments [2]. The theory accounts for the interaction between the solid and fluid phases. The sound wave propagation is governed by two coupled wave equations.

$$
\begin{gathered}
\mu \nabla^{2} u+(H-\mu) \nabla(\nabla \cdot u)-C \nabla(\nabla \bullet w)=\rho \frac{\partial^{2}}{\partial t^{2}} u-\rho \frac{\partial^{2}}{\partial t^{2}} w \\
C \nabla(\nabla \cdot u)-M \nabla(\nabla \bullet w)=\rho_{f} \frac{\partial^{2}}{\partial t^{2}} u-\frac{c \rho_{f}}{\beta} \frac{\partial^{2}}{\partial t^{2}} w-\frac{F \eta}{\kappa} \frac{\partial}{\partial t} w \\
\rho=\rho_{s}(1-\beta)+\rho_{f} \beta
\end{gathered}
$$

The terms $u$ and $w$ refer to solid and fluid particle displacements as illustrated in Fig. $1 ; \rho$ is the bulk density of medium, $\rho_{s}$ and $\rho_{f}$ the solid and fluid densities, $\beta$ the porosity; $H, C, M$ and $\mu$ are elastic moduli of the solid phase as defined in Ref. [2]; $\eta$, 
is the viscosity of the pore fluid; $\kappa, F$ and $c$ are the permeability, high-frequency correction function and tortuosity of the pore spaces.

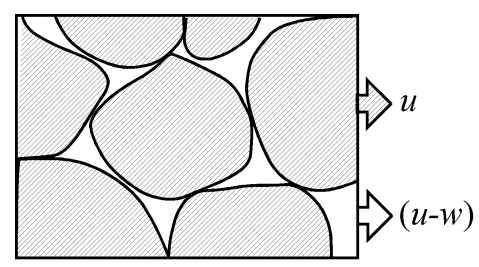

FIGURE 1. Illustration of particle displacements of the solid and fluid phases.

The Biot-Stoll model predicts two compressional waves, usually called the fast and slow waves. In ocean sediments, the fast wave is commonly identified with the sound wave. The slow wave is predicted to be highly attenuated, but its influence is indirectly evident in the reflection coefficient. The Biot-Stoll model predicts two frequency regimes, separated by the critical frequency [3], defined as,

$$
f_{c}=\frac{\beta \eta}{\kappa \rho_{f}}
$$

Below the critical frequency, the attenuation of both the sound and shear waves increase with the second power of frequency. Above this frequency, the attenuations continue to increase at a lower rate proportional to the half-power of frequency. The wave speeds show the greatest dispersion in the vicinity of the critical frequency, as illustrated in Fig. 2.
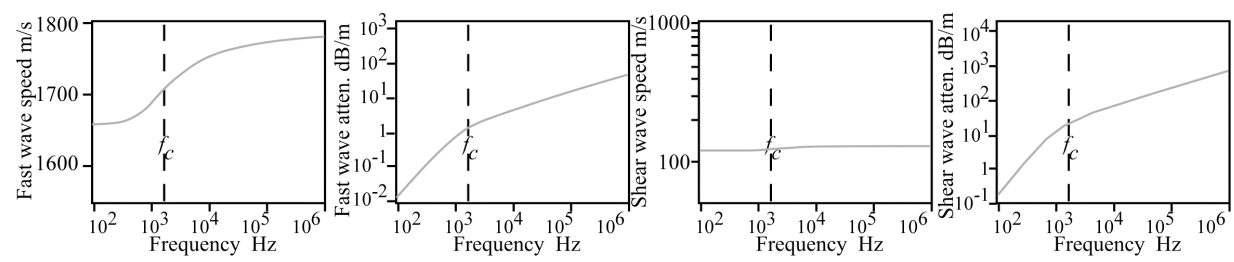

FIGURE 2. Example of sound and shear wave speeds and attenuations predicted by the Biot-Stoll model.

\section{THEORETICAL MODELS}

The frequency dependencies illustrated in Fig. 2 are not in total agreement with observations. The low-frequency dependence of attenuation on the second power of frequency is observed but not the high-frequency half-power dependence. The observed sound speed dispersion is also somewhat greater than the model prediction. To address these issues, an extension of the Biot-Stoll model, which includes the effects of contact squirt flow and viscous shear drag at the grain-grain contact (BICSQS), was developed [4]. The surface of the sand grain is rough to some degree, 
and mechanical contact is made at just a few asperities, as illustrated in the second panel in Fig. 3. These contact points are surrounded by a thin fluid film which is quite large in comparison with the solid contact area. The geometry is idealized in the third panel as a thin fluid film of uniform thickness surrounding a small central contact point. The hypothesis is that the mechanical properties of the contact are governed by both the elasticity of solid contact and the hydrodynamics of the fluid film.

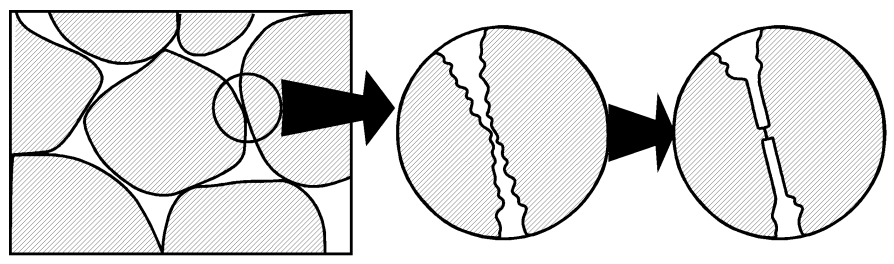

FIGURE 3. Illustration of the grain-grain contact model in the BICSQS model.

The normal $S_{n}$ and shear $S_{t}$ contact stiffnesses contain both a solid elastic component and a viscous fluid component.

$$
S_{n}=k_{c}+\frac{c_{y}}{1+i \frac{f_{c}}{f}} \quad ; S_{t}=g_{c}+i g_{c} \frac{f}{f_{\mu}}
$$

where $k_{c}$ and $g_{c}$ are the low frequency stiffnesses due to the solid connection; $c_{y}$ is the high-frequency increment due to the fluid film; $f_{c}$ and $f_{\mu}$ are the relaxation frequencies determined by the average dimensions of the fluid film. Since the elastic properties of the skeletal frame are dominated by the contact stiffnesses, its frame bulk $K_{b}$ and shear $\mu$ moduli are predicted to have the same form,

$$
K_{b}=K_{c}+\frac{C_{y}}{1+i \frac{f_{k}}{f}} ; \mu=G_{c}\left(1-i \frac{f}{f_{\mu}}\right)
$$

The terms $K_{c}$ and $G_{c}$ are the low-frequency asymptotic values due to the solid part of the contacts only; $C_{y}$ is the asymptotic increment at high frequencies. The frame shear modulus $\mu$ appears directly in Eqs. (1) and (2), and the frame bulk modulus $K_{b}$ determines the values of the other elastic coefficients $C, H$ and $M$. A comparison of the BICSQS model predictions to those of the Biot-Stoll model are shown in Fig. 4. The parameter values are from Table IV under the WJTTS column in Ref. [4].
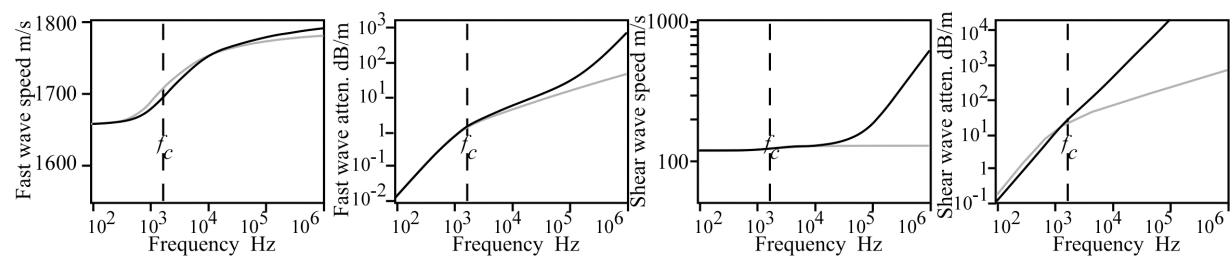

FIGURE 4. Example of wave speeds and attenuations predicted by the BICSQS model compared to the corresponding Biot-Stoll model. 


\section{EXPERMIMENTAL DATA}

The models are compared with the measured sound speed and attenuation from the Sediment Acoustics Experiments of 1999 and 2004 (SAX99 and SAX04), and the Shallow Water Experiment of 2006 (SW06), as shown in Fig. 5. The BICSQS and Biot-Stoll models predict an attenuation that increases as the second power of frequency which is in agreement with the sediment attenuation inverted from propagation measurements in SW06 [5]. The BICSQS model is also consistent with direct higher frequency attenuation measurements from the SAX99 experiment in Ref. [6]. With respect to sound speed, both models show some degree of dispersion and are consistent with the measurements above about $10 \mathrm{kHz}$. However, at low frequencies, none of the models are able to give sound speed values that are low enough to match the measured values. The measured sound speeds shown are from a number of investigators at SAX99 [6] and from Hines, Osler, Scrutton and Lyons at SAX04 [7]. The Wood's Equation sound speed, which is the sound speed in a suspension of the same porosity, is the lower bound for all effective medium models. There are data points below the Wood's Equation lower bound from both SAX99 and SAX04, as shown in Fig. 5, that need to be addressed.
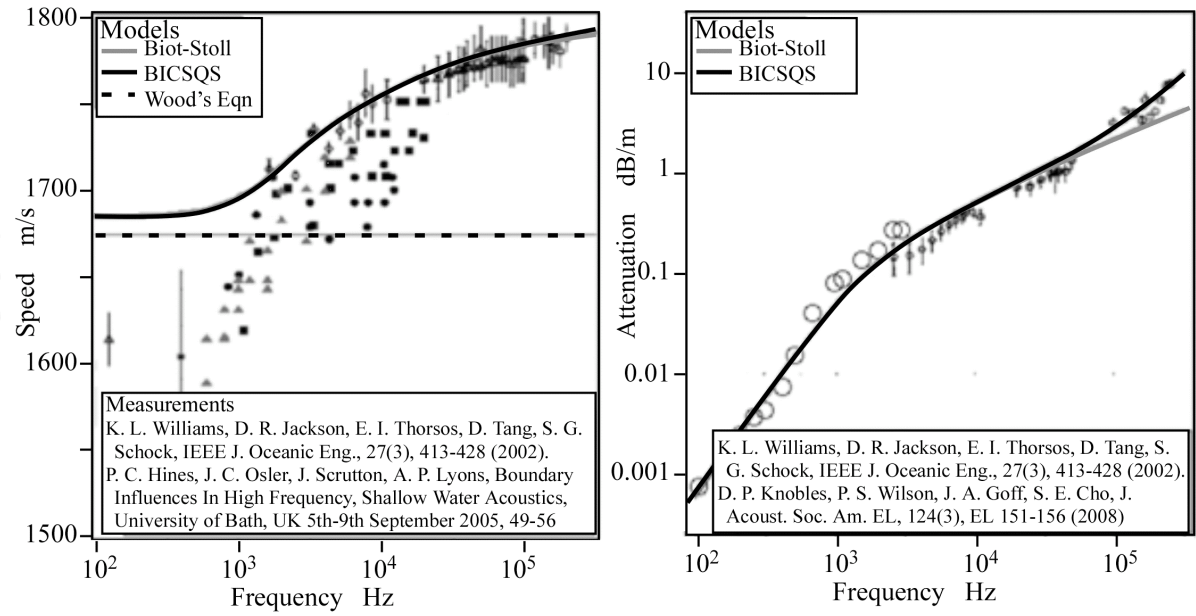

FIGURE 5. Comparison of measured sediment sound speeds from Refs. [6] and [7] and measured attenuations from [5] and [6] to the model predictions.

\section{SOUND SPEED REDUCTION IN GRANULAR MEDIA}

At this point it is instructive to review the modeling of sound speed in granular media. Given a plane acoustic wave of the form,

$$
u=A_{u} \cos \left(\frac{\omega x}{c_{p}}-\omega t\right)
$$

the potential and kinetic energies in the wave are given by, 


$$
\begin{gathered}
E_{\text {potential }}=-\left(K+\frac{4}{3} G\right) \frac{u}{2} \frac{d^{2} u}{d x^{2}}=\left(K+\frac{4}{3} G\right) \frac{\omega^{2}}{2 c_{p}^{2}} A_{u}^{2} \cos ^{2}\left(\frac{\omega x}{c_{p}}-\omega t\right) \\
E_{\text {kinetic }}=\frac{\rho}{2}\left(\frac{d u}{d t}\right)^{2}=\frac{\rho}{2} \omega^{2} A_{u}^{2} \sin ^{2}\left(\frac{\omega x}{c_{p}}-\omega t\right)
\end{gathered}
$$

In the lossless case, energy must be conserved. Therefore, given the Pythagorean relationship between the squares of the sine and cosine terms, the following solution is obtained for the sound speed,

$$
c_{p}^{2}=\frac{K+\frac{4}{3} G}{\rho}
$$

In the case of a granular medium, due to random variations in the grain-grain contact stiffness, there will be additional random motions at the grain level, that will partition the kinetic energy. This is illustrated with the aid of Fig. 6. Panel (a) shows 5 spherical grains in their initial positions. Consider the grains within the pressure peak in a plane acoustic wave traveling from left to right. In certain effective medium models, such as the Biot-Stoll, uniform deformation is assumed, which implies that spherical grains become oval and the porosity remains constant as illustrated in (b). It is obvious that uniform deformation is a poor approximation for a granular medium because most of the strain takes place at the grain-grain contact, in accordance with the Hertz-Mindlin theory. The contacts are more or less uniformly distributed over the surface of each sphere due to geometrical constraints. If all the contacts have equal stiffness, then the contact forces are symmetric about a vertical line through the center of each sphere, as illustrated in (c). However, since the packing is random, it should follow that the contact stiffnesses must also be variable and there will an asymmetry of contact forces. This will cause random rotations and translations in directions that are orthogonal to the wave vector, as illustrated in (d). Then random movements will partition the kinetic energy of the acoustic wave. Finally, some of the weaker contacts may slip and slide which tends to enhance the random motions, as illustrated in (e).

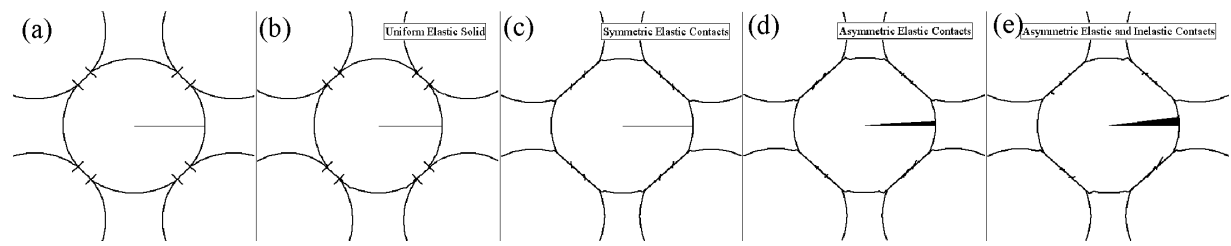

FIGURE 6. Illustration of different models of deformation in a granular medium (a) grains in their initial positions (b) uniform deformation, (c) symmetric elastic contacts, (d) asymmetric elastic contacts, and (e) asymmetric contacts with slip, in response to compression by a sound wave in the horizontal direction.

Consequently, the expression for sound speed will have an additional inertial term to account for the grain level energy due to random motion. This term will be referred to as the frame virtual mass, $m_{p}$. 


$$
c_{p}^{2}=\frac{K+\frac{4}{3} G}{\rho\left(1+m_{p}\right)}
$$

An analysis of the micromechanics [8] shows that $m_{p}$ for a dry granular medium in vacuo is given by the following expression.

$$
m_{p} \approx \frac{\sigma^{2}\left(6 N\left[R_{n t}-1\right]^{2}+50 N_{s} R_{n t}^{2}\right)}{5 N^{2}\left(2 R_{n t}+1\right)^{2}}
$$

where $\sigma^{2}$ is the scintillation index of the grain-grain contact stiffness, $N$ the coordination number, $N_{s}$ is the average number of contacts that do not slip, and $R_{n t}$ is the ratio of the tangential to normal contact stiffness. For quartz grains, $R_{n t}$ is 0.96 , or almost equal to 1. In a random close-packing of spheres of equal size, $N$ is approximately 9 [8]. It is postulated that the value of $N_{s}$ is about half of $N$ [9]. Finally, $\sigma^{2}$ is estimated to be 1 since contact stiffness is estimated to follow an exponential distribution [10].

In a water-saturated granular medium, the grain-grain contact stiffness has two components, solid and fluid, as quantified in Eq. (5). The solid component is as described above, and is expected to have a scintillation index of 1 . The fluid component, which is due to the fluid film surrounding the solid contact, is expected to have a negligible scintillation index since fluid films have a tendency to redistribute and smooth out stress concentrations. Thus, the frame virtual mass of water-saturated sand $m_{p s a t}$ is frequency dependent, as illustrated in Fig. 7, and it modifies the density of the solid frame [11].

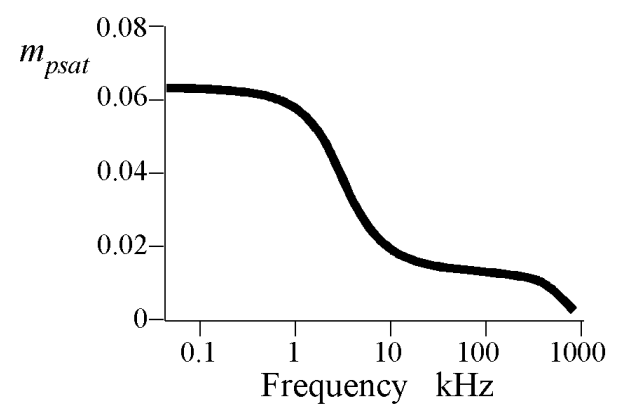

FIGURE 7. Illustration of the frame virtual mass of water-saturated sand.

At high frequencies, where the fluid component dominates, the effective value of $m_{\text {psat }}$ is negligible. At low frequencies, where the solid component is dominant, the effective value of $m_{p s a t}$ will be as given above for $m_{p}$, causing the effective inertia of the medium to be larger than the physical density, and allowing the sound speed to be lower than the Wood's Equation lower bound. Replacing $\rho_{s}$ with $\rho_{s}\left(1+m_{p s a t}\right)$ in Eq. (3), the resulting sound speed and attenuation curves from the modified BICSQS model are as shown in Fig. 8. 

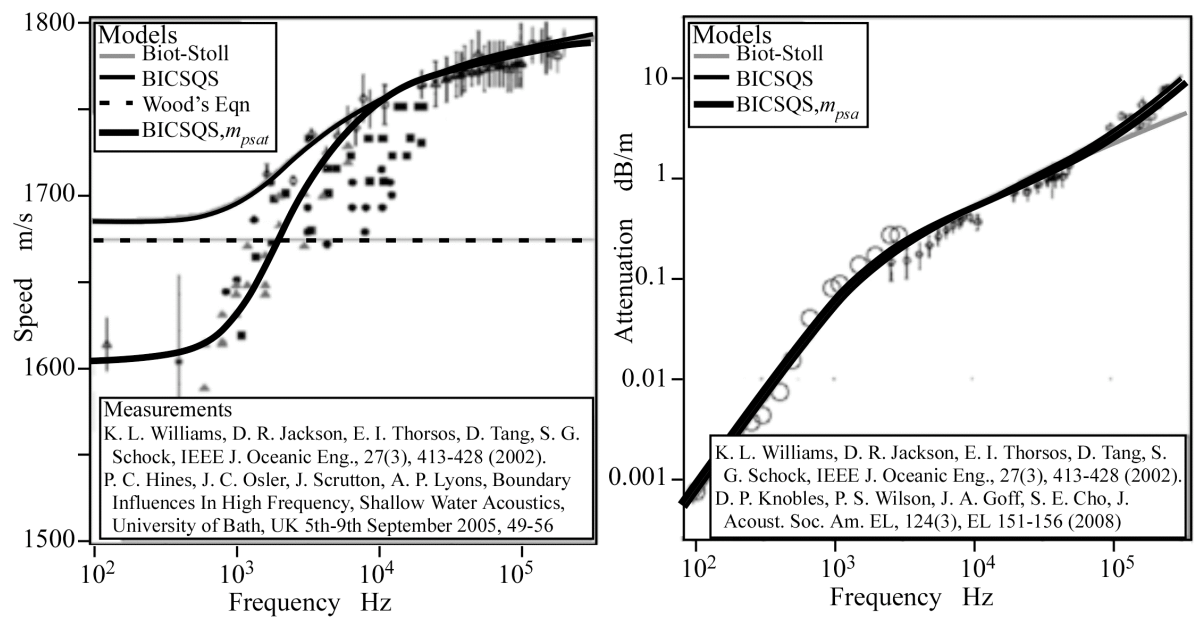

FIGURE 8. Comparison of measured sediment sound speeds from Refs. [6] and [7] and measured attenuations from [5] and [6] to the model predictions.

\section{CONCLUSIONS}

The observed low frequency attenuation in the sediment follows the second power of frequency consistent with the Biot-Stoll poroelastic model, but the observed sound speed dispersion requires a more complicated approach. The sound speed dispersion is due to three mechanisms: a relaxation process inherent in the Biot-Stoll model related to the pore fluid flow, a relaxation process due to the squirt flow of the fluid film at the grain-grain contacts, and the frequency dependence of the frame virtual mass. In conclusion, a poroelastic model that has been extended to include grain-grain contact physics, specifically squirt flow and viscous shear drag, and the micromechanical inertial effects due to randomness in the contact stiffness and the corresponding grain movement, fits well with the published experimental measurements.

\section{ACKNOWLEDGMENTS}

This work was sponsored by the United States Office of Naval Research, Ocean Acoustics Program.

\section{REFERENCES}

1. M. A. Biot, "Theory of Propagation of Elastic Waves in a Fluid-Saturated Porous Solid -- I. Low Frequency Range," J. Acoust. Soc. Am., 28(2), 168-178 (1956).

2. R. D. Stoll, Sediment acoustics, New York, Springer-Verlag, 1989.

3. T. Yamamoto, "Acoustic propagation in the ocean with a poro-elastic bottom," J. Acoust. Soc. Am., 73(5), 1587-1596 (1983).

4. N. P. Chotiros, M. J. Isakson, "A broadband model of sandy ocean sediments: Biot-Stoll with contact squirt flow and shear drag," J. Acoust. Soc. Am., 116(4), 2011-2022 (2004). 
5. D. P. Knobles, P. S. Wilson, J. A. Goff, S. E. Cho, "Seabed acoustics of a sand ridge on the New Jersey continental shelf," J. Acoust. Soc. Am. EL, 124(3), EL 151-156 (2008).

6. K. L. Williams, D. R. Jackson, E. I. Thorsos, D. Tang, S. G. Schock, "Comparison of sound speed and attenuation measured in a sandy sediment to predictions based on the Biot Theory of porous media," IEEE J. Oceanic Eng., 27(3), 413-428 (2002).

7. P. C. Hines, J. C. Osler, J. Scrutton, A. P. Lyons, "Time-of-flight measurements of acoustic wave speed in sandy sediments from $0.6-20 \mathrm{kHz}$," Boundary Influences In High Frequency, Shallow Water Acoustics, N.G.Pace and P Blondel, ( Eds), University of Bath, UK 5th-9th September 2005, 49-56 (2005).

8. G. Mavko, T. Mukerji, J. Dvorkin, The rock physics handbook, Cambridge, United Kingdom, Cambridge University Press, 1998.

9. R. Bachrach, J. Dvorkin, A. Nur, "Seismic velocities and Poisson's ratio of shallow unconsolidated sands," Geophysics, 65(2), 559-564 (2000)

10.C. H. Liu, S. R. Nagel, D. A. Schecter, S. N. Coppersmith, S. Majumdar, O. Narayan, T. A. Witten, "Force fluctuations in bead packs," Science, 269( ), 513-515 (1995).

11.N. P. Chotiros, "Wave speed dispersion in Fluid-Saturated Granular Media Due to Grain Contact Physics," Poromechanics IV: Proceedings of the Fourth Biot Conference on Poromechanics, H. I. Ling, A. Smyth, R. Betti Eds., 8-10 June 2009, Columbia University, New York, 455-460 (2009) 